\title{
Study of Arsenic Doped CdSeTe Solar Cells Using Transmission Electron Microscopy
}

Jinglong Guo ${ }^{1}$, Abhinav Sharma ${ }^{2}$, Amit Munshi $^{3}$, Carey Reich ${ }^{3}$, Adam Danielson $^{3}$, Walajabad Sampath ${ }^{3}$, Santosh Swain ${ }^{4}$ and Robert Klie ${ }^{1}$

${ }^{1}$ University of Illinois at Chicago, Chicago, Illinois, United States, ${ }^{2}$ University of New South Wales, Sydney, New South Wales, Australia, ${ }^{3}$ Colorado State University, Fort Collins, Colorado, United States, ${ }^{4}$ Washington State University, Pullman, Washington, United States

CdTe-based solar cells have shown promising increase in efficiency in the past few years. Yet the record laboratory efficiencies of CdTe-based solar cells ( 22\%) are still well below their theoretical efficiency limit of 30\%.[1] Relatively short minority carrier lifetime, low minority carrier concentrations and open circuit voltage $\left(\mathrm{V}_{\mathrm{oc}}\right)$ are believed limiting CdTe-based solar cell performance.[2] To increase the cell efficiency beyond $25 \%$ will require a Voc $>950 \mathrm{mV}$, a carrier lifetime of more than $100 \mathrm{~ns}$ and a carrier concentration of $\sim 10^{16} \mathrm{~cm}^{-3}$.

The incorporation of $\mathrm{Se}$ in the poly-crystalline CdTe absorber layers has increased the minority carrier lifetime by passivating grain boundaries and dislocations, which have been suggested to be a reason for limiting the record conversion efficiency. [3][4]Recent studies have also focused on in-situ group-V, such as As, doping of $\mathrm{CdSeTe}$, which are believed to be more stable than the commonly used $\mathrm{Cu}$ doping. As doping is expected to limit the efforts of dopant segregation towards grain boundaries, which can cause shunts and decrease in device lifetimes.[5] Metzger et al. [6] have recently demonstrated CdSeTe solar cells with group $\mathrm{V}$ doping that can reach $20.8 \%$ efficiency with an increased carrier concentration of $10^{16} \mathrm{~cm}^{-3}$.

To reach an open-circuit voltage of $1 \mathrm{~V}$, net acceptor density needs to be larger than $10^{16} \mathrm{~cm}^{-3}$.[2] In-situ As doping with $1.5 \%$ activation rate requires a very high concentration of dopants, with uniform distribution and incorporation. The absorber morphology has been reported to change with increasing As concentration, resulting in defects formation, As clustering and segregation, yet the underlying reasons are yet to be fully understood at both macro and micro scales.

In this work, we examine CdSeTe solar cell morphology changes and the formation of atomic-scale defects using the JEOL ARM200CF aberration-corrected scanning transmission electron microscope (STEM) at an acceleration voltage of $200 \mathrm{kV}$. Cross-sectional TEM samples were prepared from the as-grown samples by Focused Ion Beam (FIB).

Figure 1 shows a low-magnification low angle annular dark field (LAADF) STEM images of the $4 \mu \mathrm{m}$ thick poly-crystalline $\mathrm{CdSe}_{\mathrm{x}} \mathrm{Te}_{1-\mathrm{x}}$ films with no Arsenic incorporation (Figure 1a), as well as $10^{18} \mathrm{~cm}^{-3}$ and $10^{20} \mathrm{~cm}^{-3}$ of As concentration in Figure 1b) and c), respectively. The CdSeTe absorber layer is on top of the transparent conductive oxide (TCO) layer and glass support. We find that with the increase in As concentration, the average grain sizes decreases while the densities of stacking fault/twinning defects increase. Image post-processing was used to quantify average grain sizes of each sample. Average grain sizes of the samples shown in Figure 1, to be $1.74 \mu \mathrm{m}^{2}, 0.87 \mu \mathrm{m}^{2}$ and $0.22 \mu \mathrm{m}^{2}$, respectively. Corelating grain centers of mass indicated by red dots with grain sizes, we found near front interface As doped sample show smaller grains. Typical atomic defects structures are shown in Figure 3. 
In this contribution we will correlate how the increase of stacking fault and grain boundaries affects the device performance and explore the stability of the As dopants at the grain boundaries and front/backcontact hetero-interfaces. [7]

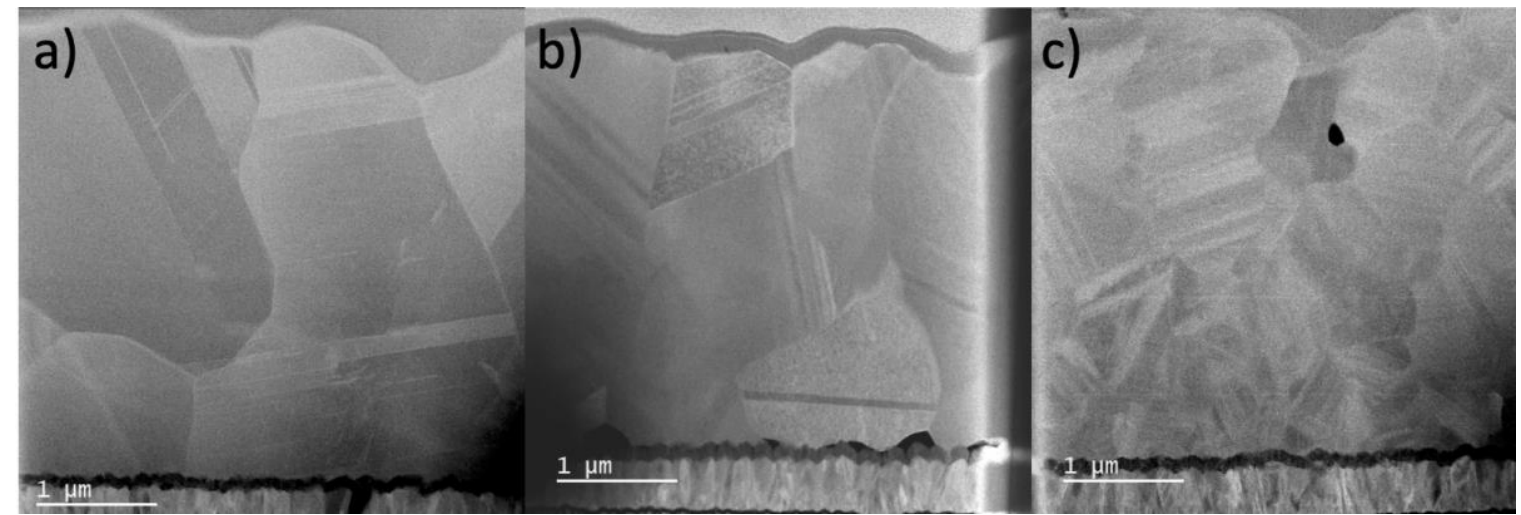

Figure 1. Low-angle annular dark-field images of samples with As concentrations of zero, $1018 \mathrm{~cm}-3$ and $1020 \mathrm{~cm}-3$, respectively.

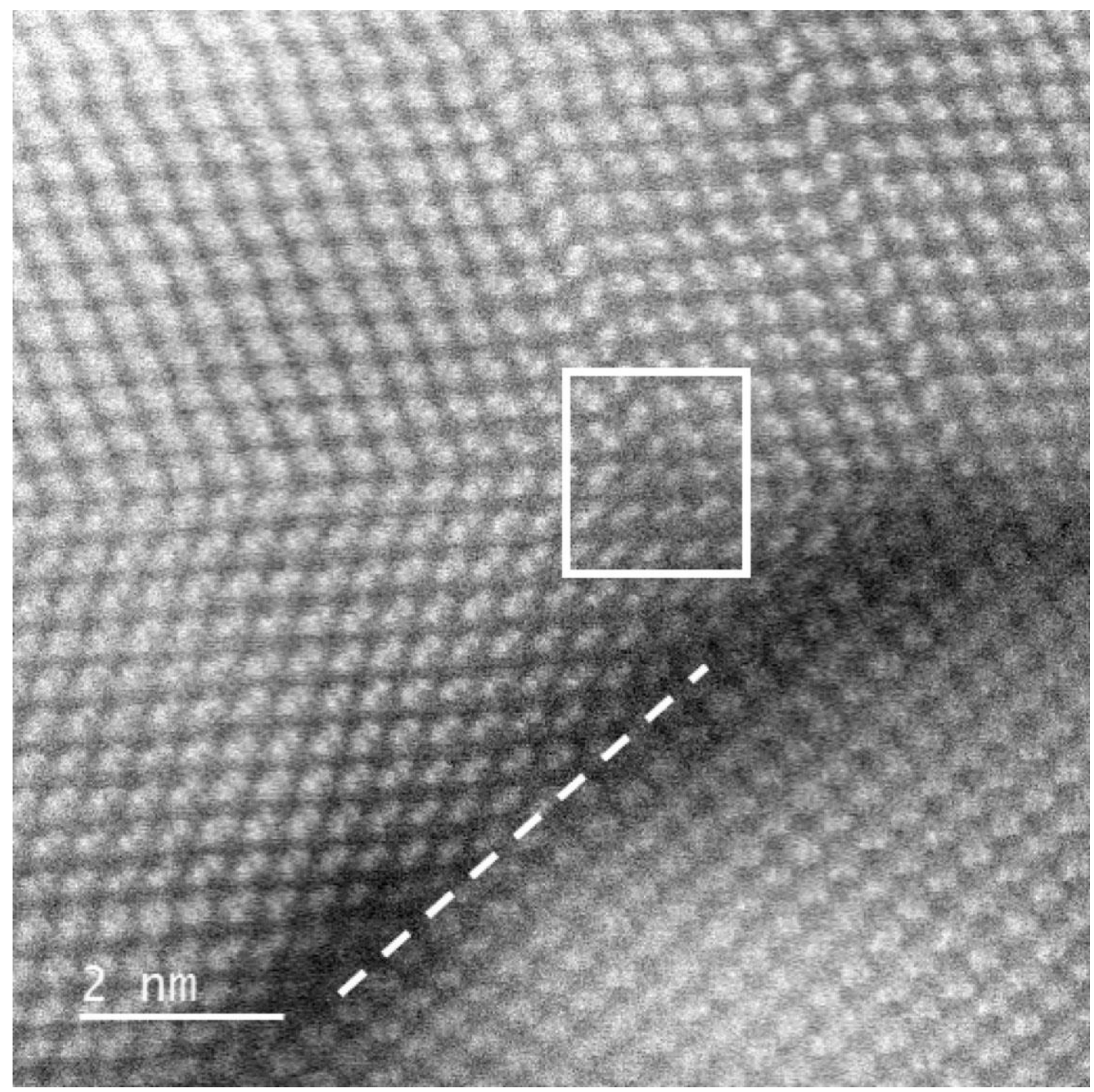


Figure 2. Atomic resolution HAADF image of cross-section specimen with As concentration of $1020 \mathrm{~cm}-$ 3. Intra-grain dislocation core is marked in white square, one grain boundary is marked with dash line.

\section{References}

[1] W. Shockley and H. J. Queisser, "Detailed balance limit of efficiency of p-n junction solar cells," J. Appl. Phys., vol. 32, no. 3, pp. 510-519, 1961.

[2] J. Sites and J. Pan, "Strategies to increase CdTe solar-cell voltage," Thin Solid Films, vol. 515, no. 15 SPEC. ISS., pp. 6099-6102, 2007.

[3] J. Guo et al., "Effect of selenium and chlorine co-passivation in polycrystalline CdSeTe devices," Appl. Phys. Lett., 2019.

[4] A. H. Munshi et al., "Polycrystalline CdSeTe/CdTe absorber cells with $28 \mathrm{~mA} / \mathrm{cm} 2$ short-circuit current," IEEE J. Photovoltaics, vol. 8, no. 1, pp. 310-314, Jan. 2018.

[5] B. E. McCandless et al., "Overcoming Carrier Concentration Limits in Polycrystalline CdTe Thin Films with In Situ Doping," Sci. Rep., vol. 8, no. 1, pp. 1-13, 2018.

[6] W. K. Metzger et al., "Exceeding 20\% efficiency with in situ group V doping in polycrystalline CdTe solar cells," Nat. Energy, pp. 37-39, 2019.

[7] This work was support by a grant from the Department of Energy, Solar Energy and Technology Office (SETO) witih grant number DE-EE0008557. 